\title{
Right coronary artery chronic total occlusion in presence of anomalous origin of left coronary artery
}

\author{
Ilaria D’Angeli • Giovanni B. Pedrazzini • \\ Francesco F. Faletra
}

Received: 14 March 2010/Accepted: 25 March 2010/Published online: 4 April 2010

(C) Springer Science+Business Media, B.V. 2010

\begin{abstract}
Right sinus origin of left coronary artery is a very uncommon congenital coronary anomaly. The presence of an associated totally occluded right coronary artery represents an exceedingly rare picture. An accurate morphologic identification of anomalous arteries, by multi-detector computed tomography, is mandatory before planning any therapeutic intervention. We report an interesting case of chronic total occlusion of the right coronary artery in a young patient with anomalous left coronary artery.
\end{abstract}

Keywords Multi-detector computed tomography · Coronary arteries - Chronic total occlusion . Congenital coronary anomaly $\cdot$ Angiography

\section{Introduction}

Right sinus origin of left coronary artery is a very uncommon congenital coronary anomaly, which may be asymptomatic or occasionally detected in patients

I. D’Angeli · G. B. Pedrazzini · F. F. Faletra

Division of Cardiology, Fondazione Cardiocentro Ticino, Lugano, Switzerland

I. D’Angeli $(\bowtie)$

Department of Heart and Great Vessels "Attilio Reale", Policlinico Umberto I, Sapienza University of Rome,

Viale del Policlinico, 15500161 Rome, Italy

e-mail: ilaria.d@libero.it with myocardial ischemia due to atherosclerotic coronary artery disease. Therefore, anomalous coronary arteries are often an incidental finding during conventional coronary angiography, with an incidence of $0.3-0.8 \%$ [1].

The presence of an associated totally occluded right coronary artery represents a more and more rare picture.

In these cases, percutaneous coronary angioplasty with stent insertion may be a successful therapeutic option; however, accurate morphologic identification of anomalous arteries is mandatory before planning these interventions, in order to recognize other possible mechanisms of myocardial ischemia (e.g. vascular compression) and to choose the most appropriate type of pharmacological, percutaneous or surgical intervention. In this case, multi-detector computed tomography (MDCT) represents an important tool for the diagnosis of the congenital anomaly [2]. It allows us to have a precise picture of the anatomy to plan further interventions. MDCT is a rapid, non invasive imaging technique capable to provide high-resolution images for the assessment of the coronary arteries and heart chambers. By an automatic software, MDCT can determinate the single components of atherosclerotic plaques and the amount of calcium (calcified spots) in the totally occluded vessels (Hounsfield Units). Finally, the new generation scanners, with prospective triggering protocols and low tube voltage, give the patient a very low X-ray dose exposure [3]. 

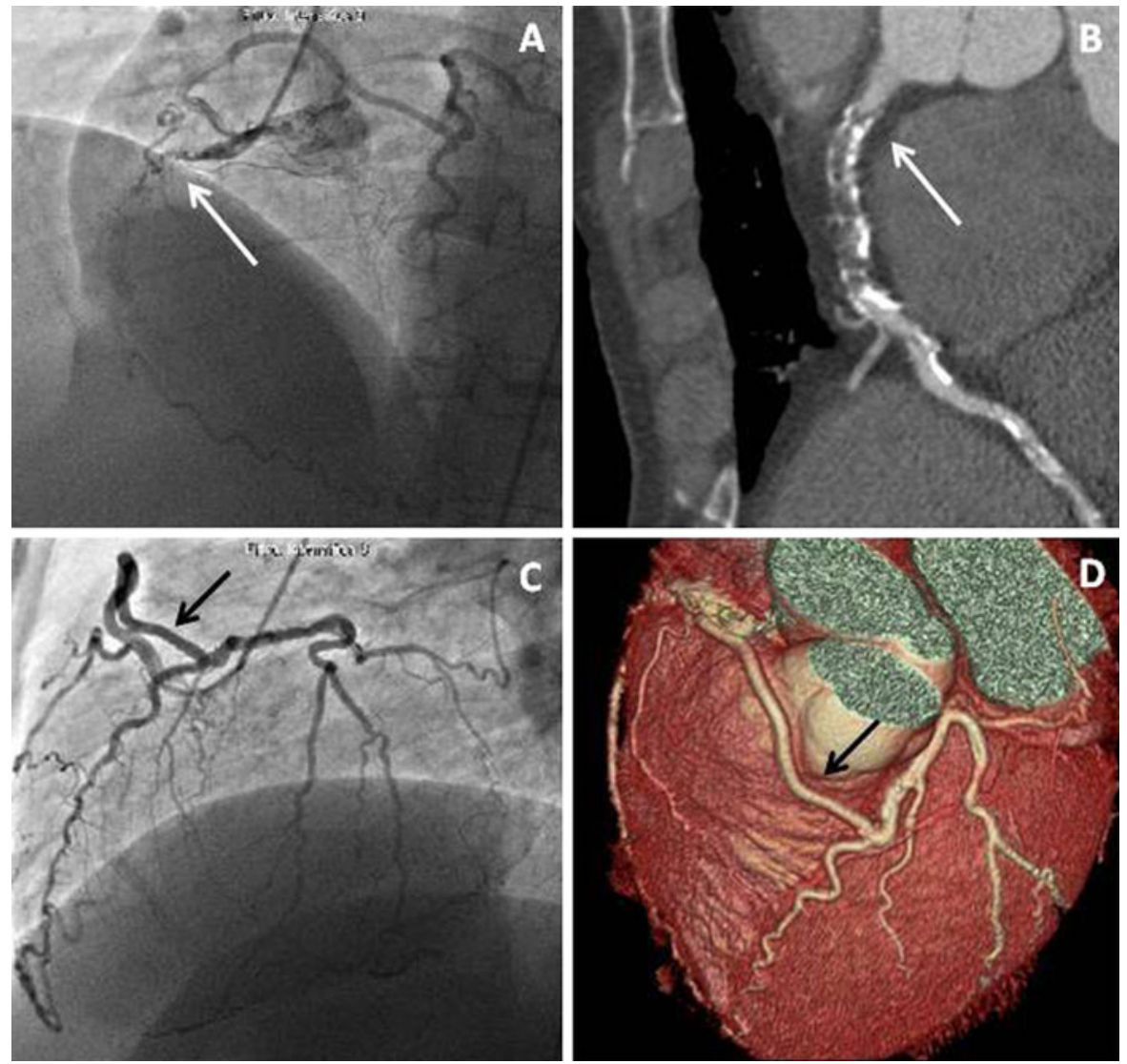

Fig. 1 Panel A, Angiography: chronic totally occluded right coronary artery. Panel B, 64-slice CT: right coronary artery occlusion with calcified spots. Panel C, Angiography: left coronary artery originating from right sinus of Valsalva. Panel

We report an interesting case of chronic total occlusion of the right coronary artery in a young patient with anomalous left coronary artery.

\section{Case report}

A 40-year-old man was admitted to hospital after several episodes of typical rest chest pain and numbness of the left arm in the past 2 months. Past medical history showed no risk factors for coronary artery disease. On first examination, blood pressure was $120 / 70 \mathrm{mmHg}$ and heart rate $75 \mathrm{bpm}$. Electrocardiography showed sinus rhythm with mild STdepression in inferior zone. Laboratory tests were in the limits of normality. An early coronary angiogram
D, 64-slice CT: 3D-volume rendering showing left coronary artery originating from right sinus of Valsalva running, with a natural graft, in front of pulmonary trunk

was performed, showing an anomalous left coronary artery arising from right sinus of Valsalva (Fig. 1, Panel C). A chronic totally occluded right coronary artery was present (Fig. 1, Panel A, white arrow). Before attempting a recanalization of the occluded vessel, a 64-slice CT scan (LightSpeed VCT, GE Healthcare, USA) was performed to evaluate the length of the occlusion and the amount of calcified spots (Fig. 1, Panel B). CT images were analyzed using multiplanar reconstruction (MPR) and curved MPR, maximum intensity projection (MIP) and 3Dvolume rendering (3DVR) techniques. Prospective triggering protocol was used. Scan parameters were: gantry rotation speed $0.35 \mathrm{~s}$, adapted tube voltage $100 \mathrm{kV}$ and effective tube current $550 \mathrm{~mA}$, because patient BMI was $<25 \mathrm{~kg} \mathrm{~m}^{2}$. The pre-selected phase 
for data acquisition was $75 \%$ of the cardiac cycle (middle of diastole). Padding was $0 \mathrm{~ms}$ because the patient had stable sinus rhythm with low heart rate. Radiation exposure was $2.05 \mathrm{mSv}$. CT scan showed the presence of left coronary artery originating from right sinus of Valsalva running, with a natural graft, in front of pulmonary trunk (Fig. 1, Panel C and D, black arrow). Chronic totally occluded right coronary artery was recanalized with the implantation of two long sirolimus-eluting stents. The procedure was performed with success and without complications, and the patient was discharged the day after in good general conditions.

\section{References}

1. Felmeden D, Singh SP, Lip GY (2000) Anomalous coronary arteries of aortic origin. Int J Clin Pract 54(6):390-394

2. Duran C, Kantarci M, Durur Subasi I, Gulbaran M, Sevimli S, Bayram E, Eren S, Karaman A, Fil F, Okur A (2006) Remarkable anatomic anomalies of coronary arteries and their clinical importance: a multi detector computed tomography study. J Comput Assist Tomogr 30(6):939-948

3. Mollet NR, Cademartiri F, van Mieghem CA, Runza G, Mc Fadden EP, Baks T, Serruys PW, Krestin GP, De Feyter PJ (2005) High-resolution spiral computed tomography coronary angiography in patients referred for diagnostic conventional coronary angiography. Circulation 112:2318-2323 